\title{
OS PROCESSOS DE SEPARAÇÃO POR MEMBRANAS E SUA UTILIZAÇÃO NO TRATAMENTO DE EFLUENTES INDUSTRIAIS DA INDÚSTRIA DE LATICÍNIOS: REVISÃO BIBLIOGRÁFICA
}

\author{
The separation processes by membrane sand their use in the \\ treatment of industrial effluents of the dairy industry: \\ literature review
}

\begin{abstract}
Douglas Felipe Galvão ${ }^{I^{*}}$, Eliane Rodrigues dos Santos Gomes ${ }^{l}$
\end{abstract}
\begin{abstract}
RESUMO
As indústrias de laticínios são grandes geradoras de efluentes em seus processos de produção. Esse efluente precisa ser tratado antes de seu lançamento em corpos hídricos, para atender aos padrões da legislação, especialmente a Resolução $n^{\circ} 357 / 2005$ do Conselho Nacional do Meio Ambiente (CONAMA) complementada e alterada pela Resolução no 430/2011(CONAMA) que "dispõe sobre condições e padrões de lançamento de efluentes". Os processos de separação por membranas têm ganhado destaque no que se refere ao tratamento de efluentes por meio de sistemas compactos de tratamento, pois possibilitam a geração de águas de reúso que podem ser reaproveitadas nos processos industriais. Nesse sentido, buscou-se na literatura estudos na área de tratamento de efluentes de laticínios por meio dos processos de separação por membranas, com o objetivo de elucidar sobre a importância desse tipo de tecnologia no tratamento de efluentes de laticínios.
\end{abstract}

Palavras-chave: leite; efluente; separação.

\begin{abstract}
Dairy industries are great generator of waste in their production processes. This effluent must be treated before its released into waterbodies, comply with AOS Legislation Standards, especially Resolution $n^{\circ} 357 / 2005$ of the National Council of the Environment (CONAMA) supplemented and amended by Resolution
\end{abstract}

1 Universidade Tecnológica Federal do Paraná, Campus Medianeira, Av. Brasil, 4232, Independência, 85884-000, Medianeira, PR, Brasil. E-mail: fgdoug@gmail.com

* Autor para correspondência.

Recebido / Received: 18/05/2016

Aprovado / Approved: 05/09/2016 
CONAMA 430/2011, which "boasts the conditions and effluent Release Standards". The membrane separation processes has gained prominence there regarding the treatment of effluents through compact treatment systems, as they allow the reuse of water generation that may be reusedin industrial processes. In this sense, we sought in the literature studies in the treatment area of dairy effluent through the membrane separation processes, with the aim of elucidating the importance of this type of technology in the treatment of dairy effluents.

Keywords: milk; effluent; separation.

\section{INTRODUÇ̃̃O}

Os processos de separação por membranas utilizam como barreira seletiva membranas sintéticas e, por meio deste processo, é possível separar, concentrar e purificar substâncias (HABERT et al., 2006). Assim, podem ser utilizados no tratamento de efluentes de laticínios, com o objetivo de melhorar a qualidade destes.

A indústria de laticínios caracteriza-se pelo elevado consumo de água. A necessidade de água ocorre, principalmente, pela manutenção das condições de limpeza, das condições sanitárias e de higiene nos setores de produção. Com o elevado consumo de água para o processamento de leite, ocorre a geração de grandes vazões de efluentes, o que torna a indústria de laticínios um potencial poluidor (MAGANHA, 2008; VOURCH et al., 2008).

Com a escassez de água e a necessidade de pagamento pela utilização desse recurso, um dos instrumentos de gestão da Política Nacional de Recursos Hídricos, instituída pela Lei $n^{\circ}$ 9.433/97 (BRASIL, 1997), é a minimização do consumo nos processos industriais que torna-se cada dia mais essencial. O uso consciente, as novas tecnologias que utilizam menos água para a realização do mesmo processo e a adoção de formas de reúso podem ser atitudes relevantes para a redução do consumo de água pelo setor industrial (MIERZWA; HESPANHOL, 2005).

Dessa forma, os processos de separação por membranas têm gerado interesse pelas indústrias de laticínios, especialmente pela possibilidade de geração de águas de reúso de boa qualidade que podem auxiliar na minimização do consumo, bem como na redução da geração de efluentes por estas indústrias.

Com o objetivo de elucidar sobre os processos de separação por membranas no tratamento de efluentes de laticínios, realizou-se pesquisa na literatura nacional e internacional, utilizando-se os estudos já publicados referentes ao tema, verificando-se resultados obtidos e a importância com relação ao reúso de água e a valorização de produtos.

\section{REFERENCIAL TEÓRICO}

\section{Efluentes da indústria de laticínios}

Os efluentes líquidos industriais são aqueles oriundos de operações e processos nos quais utiliza-se água, e corresponde a parcela não incorporada ao produto final e a parcela líquida proveniente da matéria-prima que é removida nos processos industriais (PIRES et al., 2013; CAVALCANTI, 2012).

As águas residuárias industriais variam muito, tanto em composição quanto em quantidade e são dependentes da diversidade dos produtos que são fabricados, da capacidade produtiva da indústria, da modernidade dos processos empregados, da natureza da atividade, de quais matériasprimas são utilizadas, além das práticas de reciclagem e do reúso que são utilizadas (CAVALCANTI, 2012). 
As características dos efluentes podem ser de biodegradabilidade, semelhante aos esgotos sanitários, ou completamente não biodegradáveis, principalmente os oriundos de indústrias de produtos metálicos como as de galvanoplastia. As indústrias alimentícias, de papel e celulose e sucroalcooleiras, caracterizam-se pela geração de efluentes biodegradáveis e ricos em matéria orgânica (PIRES et al., 2013).

Entre as indústrias alimentícias, a de laticínios está entre as mais poluentes, principalmente pelo grande consumo de água e geração de efluentes (VOURCH et al., 2008). De acordo com Saraiva et al. (2009), tal geração varia de 1,1 a $6,8 \mathrm{~m}^{3} / \mathrm{m}^{3}$ de leite processado. Tchamangoet al. (2010) citam que tal valor varia de 0,2 a 10 litros de efluente para cada litro de leite processado. $\mathrm{Na} \mathrm{Ta}-$ bela 1 observa-se o volume de geração de efluentes de acordo com as diferentes linhas de produção em um laticínio.

As características do efluente gerado variam bastante conforme o padrão e as tecnologias utilizadas na indústria de laticínios, em geral apresentam concentração elevada de matéria orgânica, quantidades consideráveis de nutrientes, poluentes orgânicos e agentes infectantes, além de resíduos de leite, proteínas, carboidratos, gorduras e resíduos de agentes de limpeza (LÁSLÓ et al., 2009; SARAIVA et al., 2009). Na Tabela 2 observa-se as características para os diferentes parâmetros do efluente bruto de laticínio, conforme Maganha (2008).

Estas águas residuárias, se não tratadas e despejadas nos recursos hídricos, poderão causar diversos problemas, tais como a mortandade das espécies aquáticas, prejuízos à saúde pública pelo consumo de água poluída e gerar aumento nos custos de tratamento deste recurso (SCHMITT et al., 2014; LIMA, 2010). De tal maneira, torna-se importante utilizar sistemas de tratamento de efluentes otimizados que sejam integrados com a identificação dos pontos de geração de despejos líquidos no processo de produção para que se possa chegar a uma produção sustentável (SARAIVA et al., 2009).

Frente ao exposto, a busca por alternativas internas de reúso industrial e a compra de efluentes tratados de companhias de saneamento a preços inferiores aos da água potável tem gerado interesse às indústrias, pois minimizam o consumo de água nos processos e, consequentemente, reduzem a geração de efluentes (CONSTANZI, 2007).

Nesse sentido, Hespanhol (2002) afirma ainda que, deve-se optar por satisfazer àquelas demandas que exigem águas de baixa qualidade por fontes menos nobres e utilizarse de fontes de qualidade superior somente para usos mais nobres, como o abastecimento doméstico.

Tabela 1 - Volume de efluente por quilograma de leite processado nas diferentes linhas de produção da indústria de laticínios

\begin{tabular}{lc}
\hline \multicolumn{1}{c}{ Tipo de Produto } & $\begin{array}{c}\text { Volume de efluentes líquidos } \\
\text { (litro/kg de leite processado) }\end{array}$ \\
\hline $\begin{array}{l}\text { Produtos "brancos" (leite, cremes e iogurtes) } \\
\text { Produtos "amarelos" (manteiga e queijos) } \\
\begin{array}{l}\text { Produtos "especiais" (concentrados de leite } \\
\text { ou produtos lácteos desidratados) }\end{array}\end{array}$ & 3 \\
\hline
\end{tabular}

Fonte: Maganha (2008). 
Tabela 2 - Caracterização dos efluentes da indústria de laticínios

\begin{tabular}{ccc}
\hline \multirow{2}{*}{ Parâmetro } & \multicolumn{2}{c}{ Faixa de Variação } \\
\cline { 2 - 3 } & $24-5700 \mathrm{mg} / \mathrm{L}$ & $100-1000 \mathrm{mg} / \mathrm{L}$ \\
Sólidos suspensos & $135-8500 \mathrm{mg} / \mathrm{L}$ & $100-2000 \mathrm{mg} / \mathrm{L}$ \\
Sólidos suspensos totais & $500-4500 \mathrm{mg} / \mathrm{L}$ & $6000 \mathrm{mg} / \mathrm{L}$ \\
DQO & $450-4790 \mathrm{mg} / \mathrm{L}$ & $4000 \mathrm{mg} / \mathrm{L}$ \\
DBO5 & $210-560 \mathrm{mg} / \mathrm{L}$ & $\mathrm{ND}$ \\
Proteína & $35-500 \mathrm{mg} / \mathrm{L}$ & $95-550 \mathrm{mg} / \mathrm{L}$ \\
Gordura/óleos e graxas & $252-931 \mathrm{mg} / \mathrm{L}$ & $\mathrm{ND}$ \\
Carboidrato & $10-100 \mathrm{mg} / \mathrm{L}$ & $\mathrm{ND}$ \\
Amônia $-\mathrm{N}$ & $15-180 \mathrm{mg} / \mathrm{L}$ & $116 \mathrm{mg} / \mathrm{L}$ \\
Nitrogênio & $20-250 \mathrm{mg} / \mathrm{L}$ & $0,1-46 \mathrm{mg} / \mathrm{L}$ \\
Fósforo & $60-807 \mathrm{mg} / \mathrm{L}$ & $\mathrm{ND}$ \\
Sódio & $48-469 \mathrm{mg} / \mathrm{L}$ & $\mathrm{ND}$ \\
Cloretos & $57-112 \mathrm{mg} / \mathrm{L}$ & $\mathrm{ND}$ \\
Cálcio & $22-49 \mathrm{mg} / \mathrm{L}$ & $\mathrm{ND}$ \\
Magnésio & $11-160 \mathrm{mg} / \mathrm{L}$ & $\mathrm{ND}$ \\
Potássio & $5,3-9,4$ & $1-12$ \\
pH & $12-40^{\circ} \mathrm{C}$ & $20-30^{\circ} \mathrm{C}$ \\
Temperatura &
\end{tabular}

Fonte: (1) Enviroment Agency of England and Wales (2000)/ European Comission - Integrated Pollution Prevent and Control (2006); (2) ABIQ; citado sem Maganha (2008).

Em resposta a tais condições, tem aumentado o desenvolvimento das tecnologias de tratamento de efluentes a fim de satisfazer os limites de qualidade para o reúso. Assim, atualmente, águas de reúso podem tornar-se uma importante contribuição para o suprimento de água na indústria (ÁREVALO et al., 2012; ANDRADE et al., 2012; ANDRADE et al., 2014; VOURCH et al., 2008).

Pode-se definir reúso como a utilização do efluente diretamente ou após alguma etapa de tratamento em outros processos, de acordo com a qualidade de água requerida. Nem toda a parcela de efluente gerada, precisa ser tratada para ser reutilizada, porém, em determinados casos, há necessidade de tratamentos específicos para purificação (TELLES; COSTA, 2010). O reúso pode ser concebido também como a utilização de efluentes tra- tados ou não para fins que sejam benéficos, citando-se a irrigação, o uso industrial e fins urbanos que não sejam potáveis (MIERZWA; HESPANHOL, 2005).

Alguns processos industriais, mais especificamente os da indústria alimentícia, não permitem a utilização de águas de reúso em seus processos de produção. Entretanto, mesmo nestas indústrias, há a possibilidade de reutilização da água em processos que exigem qualidade de água menos nobres, como para refrigeração, alimentação de caldeiras, limpeza de pisos, descargas sanitárias em banheiros, entre outros.

Devido às técnicas de tratamento existentes nas indústrias, um efluente tratado pode apresentar características físicas, químicas ou biológicas semelhantes ou até melhores que as da água bruta. Da mesma forma, efluentes 
de determinados processos apresentam qualidade adequada a outros processos, sem a necessidade de tratamento (MIERZWA; HESPANHOL, 2005).

Os processos de separação por membranas têm ganhado destaque na indústria de laticínios no sentido de gerar águas de reúso com qualidade elevada, que ampliam as possibilidades de reúso nas indústrias.

\section{Processos de separação por membranas}

Os processos de separação por membranas utilizam como barreira seletiva membranas sintéticas, nas quais tenta-se utilizar as características desejáveis das membranas naturais (biológicas), principalmente as de permeabilidade e seletividade. Não acontece nenhuma transformação química durante o tratamento por membranas, apenas o material fica retido durante a filtração (SCHNEIDER; TSUTYIA, 2001).

Tais sistemas são utilizados para separar da água substâncias e sólidos que têm pequenos diâmetros, além de moléculas e compostos iônicos, através da aplicação de algum tipo de força externa. As forças externas utilizadas na filtração em membranas podem ser a pressão, a sucção ou ainda o potencial elétrico (SCHNEIDER; TSUTYIA, 2001; HABERT et al., 2006).

Após a passagem do líquido pela membrana este passa a ser chamado de permeado e o que é retido é chamado de concentrado ou retentado (MIERZWA, 2007). A Figura 1, a seguir, apresenta o esquema simplificado de entrada e saída da solução na membrana.
Apesar de alguns estudos na área de membranas terem ocorrido desde a década de 1930, somente na década de 1950 as pesquisas com membranas passaram a gerar maior interesse, quando, nos Estados Unidos, passou-se a investir em pesquisas para a dessalinização de água do mar, ocorrendo avanços com relação as técnicas de produção das membranas e aumento do fluxo permeado de água (HABERT et al., 2006).

Mais recentemente a sua utilização aconteceu na indústria química, na década de 1970, aliada a outros tipos de processos clássicos de separação como a filtração, absorção, troca iônica e dentre outros processos (HABERT et al., 2006).

Schneider e Tsutyia (2001) afirmam ainda que, inicialmente, o tratamento de membranas tinha por finalidade a dessalinização de água do mar, utilizando-se o sistema com membranas de osmose reversa. Somente a partir dos anos 90 a tecnologia passou a ser utilizada para o tratamento de água e esgoto.

Principalmente devido à cobrança pelo uso da água aliada à necessidade de preservação do meio ambiente, aumentou o interesse pelo reúso da água. Nesse sentido, os processos de separação por membranas no tratamento de efluentes se apresentam como uma das tecnologias mais promissoras, possibilitando a reutilização das águas e, consequentemente, reduzindo o consumo de água de boa qualidade em processos que não exijam qualidade tão alta, otimizando sua utilização nos processos industriais (ANDRADE et al, 2012).

Segundo Mierzwa; Hespanhol (2005), os processos de separação por membranas

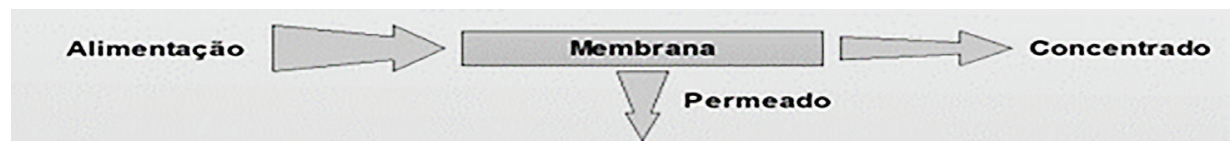

Fonte: Adaptado de Mierzwa(2007).

Figura 1 - Esquema representando a entrada do líquido, a saída do permeado e do concentrado (retentado) 
diferem dos sistemas de filtração convencional, porque o fluxo de alimentação é paralelo às membranas.

Esses processos apresentam inúmeras vantagens, tendo em vista que as membranas têm eficiência para reter partículas sólidas de dimensões pequenas e compostos orgânicos e inorgânicos dissolvidos. Além disso, a pressão do sistema de separação por membranas é maior que nos processos de filtração convencional (MIERZWA; HESPANHOL, 2005).

Os materiais utilizados na produção das membranas variam. Há membranas prepara- das com materiais poliméricos (orgânicos) e aquelas que são produzidas com materiais inorgânicos. Os materiais inorgânicos já são utilizados na produção de membranas há mais de 20 anos. Porém, ganharam maior espaço no mercado apenas recentemente (HABERT, et al., 2006). Os materiais orgânicos para produção das membranas são basicamente os polímeros e os inorgânicos são os metais e as cerâmicas.

Os polímeros mais utilizados na fabricação de membranas são expostos a seguir, na Tabela 3 .

Os processos utilizando membranas

Tabela 3 - Tipos de polímeros utilizados na produção das diferentes membranas

\begin{tabular}{cc}
\hline Membrana & Material \\
\hline Microfiltração (MF) & Cerâmica \\
& Polipropileno (PP) \\
\hline Ultrafiltração (UF) & Cerâmica \\
& Acetato de Celulose (AC) \\
& Polisulfona (PS) \\
& Polietersulfona (PES) \\
& Polivinilpirolidona \\
& Poliacrinonitrila (PAN) \\
\hline Nanofiltração (NF) & Fluoreto de polivinilideno (PVDF) \\
\hline Osmose Reversa (OR) & Poliacrilamida \\
\hline
\end{tabular}

Fonte: Adaptado de Cavalcanti (2012); Wagner (2001).

Tabela 4 - Tipo de membrana e força motriz

\begin{tabular}{lc}
\hline \multicolumn{1}{c}{ Membrana } & Força Motriz \\
\hline Microfiltração (MF) & $<2$ bar \\
Ultrafiltração (UF) & $1-10$ bar \\
Nanofiltração (NF) & $5-35$ bar \\
Osmose Reversa (OR) & Diferença de Concentração (15-150 bar) \\
Eletrodiálise & Tensão e corrente elétrica \\
\hline
\end{tabular}

Fonte: Adaptado de Cavalcanti (2012); Wagner (2001). 


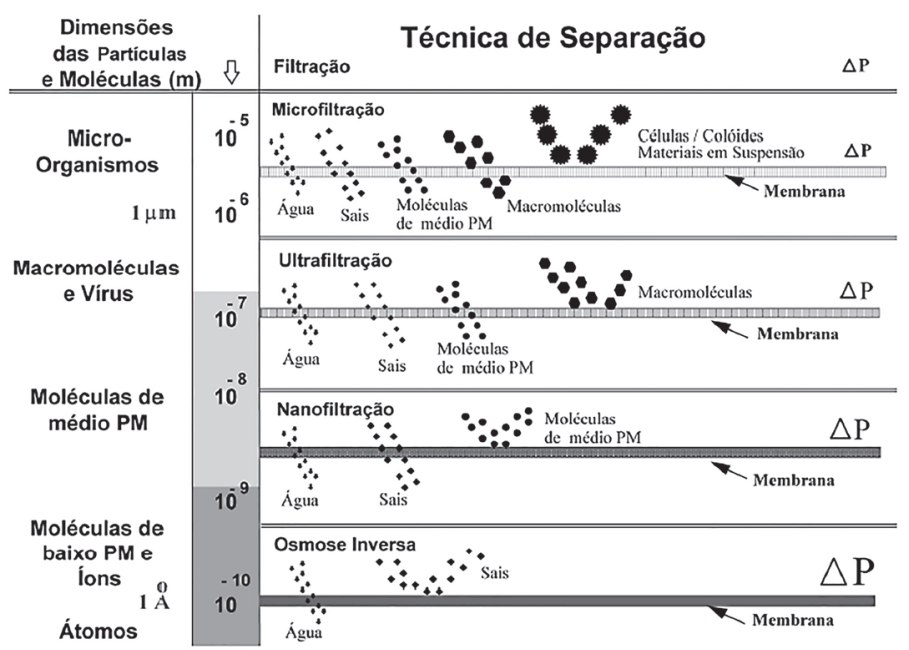

Fonte: Habert et al. (2006).

Figura 2 - Partículas e moléculas retidas de acordo com o tipo de processo de separação por membranas

são basicamente cinco: microfiltração (MF), ultrafiltração (UF), nanofiltração (NF), osmose reversa (RO) e eletrodiálise (ED) (SCHNEIDER; TSUTYIA, 2001). A diferença de cada um é a dimensão das partículas e das moléculas que são retidas e com qual intensidade a força motriz promove a separação, conforme visualizado na Figura 2 e na Tabela 4, respectivamente (HABERT, et al. 2006; CAVALCANTI, 2012; WAGNER, 2001).

Comparando-se com técnicas físicoquímicas tradicionais aos sistemas de tratamento por membranas, podem-se citar diversas vantagens, tais como necessidade de tratamento químico somente para a limpeza da membrana e alta taxa de transferência que ocorre durante o processo, mantendo a pureza do produto em condições ambientes (ACERO et al., 2010). As vantagens salientadas são importantes por reduzirem a poluição ambiental, aumentarem a segurança e a eficiência do processo.

Para a utilização dos sistemas de tra- tamento por processos de separação por membranas é necessária a utilização dos chamados módulos de membranas. De acordo com Schneider; Tsutyia (2001) o módulo é um sistema básico que possibilita a utilização das membranas como unidade de separação. A sua composição é a seguinte: membranas, estruturas de suporte de pressão (vácuo ou corrente elétrica) e canais de alimentação e remoção do concentrado e do permeado.

De acordo com Schneider; Tsutiya (2001) os principais módulos são os módulos com placas de membranas, módulos tubulares, módulos espirais, módulos com fibras ocas e módulos com discos rotatórios.

\section{Operação dos processos de separação por membranas e fatores que influenciam no fluxo}

Analisando um sistema de tratamento por membranas, Schneider; Tsutyia (2001) afirmam que, somando-se ao módulo, estes sistemas são formados por um sistema de 
pressurização para pressurizar o canal que alimenta a membrana, uma válvula no canal do concentrado que regula a pressão no canal de alimentação, um canal de coleta do permeado e os elementos de remoção do material retido na superfície.

Nos processos de separação por membranas, o fluxo permeado é independente do tempo, dependendo especificamente do potencial de pressão aplicado ou então da difusão pelo material da membrana. Essa independência do tempo, indica a estabilidade mecânica da membrana, da pureza do solvente e da sua baixa interação com o material de fabricação da membrana. Quando não há observação de uma dessas condições, o fluxo permeado irá diminuir com o tempo, indicando que ocorre alguma alteração com a membrana (HABERT et al., 2006).

Habert et al. (2006) indicam como principais causadores do decaimento do fluxo a deformação mecânica, o inchamento da matriz mecânica, a interação do solvente com o material da membrana ou então pela presença de impurezas no solvente que causa o entupimento dos poros.

Schneider; Tsutyia (2001) citam que o fluxo do líquido através da membrana é dependente de alguns fatores, como: diâmetro dos poros, porosidade da membrana (fração da área de membrana ocupada por poros), espessura da membrana, camada de concentração e polarização, tortas de filtro (material retido), fouling químico (sais precipitados ou géis) e biofilmes.

Um dos piores problemas citados pelos autores durante a operação dos sistemas de membranas é o decaimento do fluxo com o tempo. Esse fenômeno é denominado de fouling ou colmatação da membrana (LAUTENSCHLAGER et al., 2009; HASAN, et al., 2013; GIACOBBO et al., 2010; SONG, 1998; VIDAL; CAMPOS; 2009).

Essa diminuição do fluxo ocorre por dois motivos essenciais: bloqueio dos poros da membrana, que aumenta a resistência ao fluxo, e a formação de uma camada de incrustação a qual cria uma camada adicional de resistência ao fluxo permeado. Outros fatores como adsorção do soluto, depósito de partículas dentro dos poros da membrana e mudança nas características da camada de incrustação podem afetar o fouling da membrana ou aumenta-lo, ou ainda modificar ambos os fenômenos essenciais do fouling (entupimento dos poros e formação da camada de incrustação). A formação da camada de concentração de polarização pode também acrescentar uma camada de resistência (SONG, 1998).

De acordo com Giacobbo et al. (2010), o fouling refere-se ao acúmulo de contaminantes na superfície ou dentro dos poros da membrana, ocasionando a diminuição do fluxo permeado. Já o fenômeno de polarização por concentração é o aumento da concentração de soluto na interface membrana/solução, o que acaba gerando uma retrodifusão do soluto em direção ao seio da solução. Ou seja, o soluto fica concentrado na região próxima da interface membrana/solução impedindo a passagem do solvente.

Segundo os autores Lautenschlager et al. (2009), a redução no fluxo permeado durante a filtração, aplicando-se pressão constante, ocorre devido a deposição de partículas coloidais e macromoléculas na superfície da membrana. Dessa forma, os autores afirmam que a limpeza da membrana deve ser realizada periodicamente durante a operação do sistema, para prevenir a possibilidade de colmatação extrema da membrana e até mesmo sua colmatação irreversível.

O fouling acaba aumentando a complexidade de operação dos sistemas de separação por membranas, pois o sistema precisa ser parado frequentemente para restabelecimento do fluxo através da retrolavagem, resultando em aumento dos custos econômicos e tornando menos viáveis a utilização de 
sistemas de UF e MF para muitos processos (SONG, 1998).

As formas de limpeza das membranas podem ser físicas e químicas. As físicas são dependentes de forças mecânicas para remoção das partículas acumuladas na superfície da membrana. Incluem a lavagem na direção do fluxo (flushing) e/ou flushing reverso e retrolavagem (LAUTENSCHLAGER et al., 2009).

Os métodos químicos dependem de reações químicas, que quebram as forças que ligam as substâncias aderidas na superfície da membrana. As formas de limpeza química são a hidrólise, digestão, saponificação, solubilização e dispersão (LAUTENSCHLAGER et al., 2009).

\section{Processos de separação por membranas no tratamento de efluentes de laticínios}

A utilização de tratamentos para purificação da água e possibilidade de reúso, auxiliam na diminuição do total de água consumida e a produção de efluentes durante os processos utilizados na indústria de laticínios(BALANNEC, 2005).

Baldasso (2008) utilizou as membranas para separar e fracionar proteínas específicas encontradas no soro de leite. O soro é descartado no tratamento de efluentes de laticínios onde há principalmente produção de queijo e é responsável por grande poluição, especialmente pela grande quantidade de matéria orgânica. Utilizando membranas de ultrafiltração, a autora encontrou grande possibilidade para o fracionamento das proteínas, o que é extremamente importante para as indústrias, especialmente na indústria de laticínios.

Utilizando vapor condensado de indústria de laticínio, Chmiel et al. (2000) realizaram a nanofiltraçãono tratamento desta água residuária, conseguindo manter todos os parâmetros requeridos para a utilização como água de reúso nas caldeiras. De acordo com os autores, o reúso de água nas caldeiras é importante para as indústrias, pois nestes processos o volume de água utilizado é considerável. O reúso permite a minimização do desperdício da água.

Khider et al. (2004) utilizaram uma membrana de ultrafiltração comercial e uma membrana de ultrafiltração com suporte de argila, no estudo da remoção de Demanda Bioquímica de Oxigênio (DBO) e Demanda Química de Oxigênio (DQO) de efluente de laticínio. As amostras de efluente bruto foram filtradas e conseguiram a remoção de $4000 \mathrm{mg} / \mathrm{L}$ e $1800 \mathrm{mg} / \mathrm{L}$ de DBO e DQO, respectivamente, para 90 e $62 \mathrm{mg} / \mathrm{L}$ com a membrana comercial. Para a membrana com suporte de argila, as características do permeado chegaram a $60 \mathrm{mg} / \mathrm{L}$ para $\mathrm{DBO}$ e $42 \mathrm{mg} / \mathrm{L}$ para DQO. Como a DBO e a DQO representam a carga orgânica do efluente, a remoção de tais parâmetros é essencial para a qualidade do efluente a ser lançado.

Nos processos de separação por membranas, além do efluente tratado ser reutilizado como águas de reúso dentro dos processos, há a possibilidade de concentração do material retido pela membrana e sua reutilização na fabricação de novos produtos. Isso permite a valorização dos recursos da indústria.

Estudos como o de Brião e Tavares (2007) que utilizaram a ultrafiltração como processo de tratamento e reutilização de efluentes de laticínios, encontraram a possibilidade da reutilização do permeado na limpeza de equipamentos e do concentrado, após pasteurização, na produção de subprodutos lácteos como o doce de leite.

A utilização da coagulação associada a processos de separação por membranas permitem elevadas remoções de contaminantes dos efluentes. Nesse sentido, Schimitt et al. (2013) utilizaram efluente oriundo de processo de limpeza de pasteurizadores de uma indústria de laticínios. A membrana de UF 
utilizada era de cerâmica, com poro de $0,1 \mu \mathrm{m}$ e retenção molar de $4 \mathrm{kDa}$. Estes autores encontraram remoções de DQO e de turbidez acima de $96 \%$ para os tratamentos utilizando UF associada a coagulação por Moringa oleifera.

\section{CONSIDERAÇÕES FINAIS}

Os processos de separação por membranas têm sido estudados e utilizados na indústria de laticínios, principalmente visando o tratamento de efluentes para geração de águas de reúso, o que acaba por contribuir para a preservação do recurso água, gera economia para indústrias e contribui para a sustentabilidade.

Os resultados explorados demonstram que os processos de separação por membranas apresentam eficiência considerável na remoção de matéria orgânica do efluente da indústria de laticínios, o que é relevante considerando a alta taxa orgânica que compõe os resíduos líquidos gerados nos processos.

As possibilidades de estudo se ampliam em se tratando de membranas, pois há a possibilidade de reutilização do efluente permeado (tratado) nos processos industriais e a do concentrado gerado pela membrana na fabricação de subprodutos, por exemplo. Tal possibilidade é importante para as indústrias, pois pode possibilitar a minimização dos custos de produção e, consequentemente, do preço do produto, tornando a indústria mais competitiva no mercado.

\section{REFERÊNCIAS}

ACERO, J. L. et al. Membrane filtration technologies applied to municipal secondary effluents for potencial reuse. Journal of Hazardous Materials, v. 177, p. 390-398, 2010 .

ANDRADE, L. H. et al. Nano filtration as a post treatment to membrane bioreactor effluent for dairy waste water reuse. Procedia Engineering, v. 44, p. 1956-1960, 2012.

ANDRADE, L. H. Distribuição de massa molar em um biorreator com membrana para tratamento de efluente de laticínios. Revista de Engenharia Sanitária e Ambiental, v. 19, n. 3 , p. $325-334,2014$

ÁREVALO, J. et al. Waste water reuse after treatment by MBR. Microfiltration or ultrafiltration? Desalination Journal, v. 299, p. 22-27, 2012.

BALANNEC, B. Comparative Study of different nano filtration and reverse osmosis membranes for dairy effluent treatment by dead-end filtration. Separation, Purification and Technology, n. 42, p. 195-200, 2005.

BALDASSO, C. Concentração, purificação e fracionamento das proteínas do Soro Lácteo através da Tecnologia de Separação por Membranas. Dissertação (Mestrado em Engenharia Química) - Universidade Federal do Rio Grande do Sul, Porto Alegre, 2008.

BRASIL. Lei $n^{\circ} 9.433$ de 8 de janeiro de 1997. Institui a Política Nacional de Recursos Hídricos, cria o Sistema Nacional de Gerenciamento de Recursos Hídricos, Brasília, DF, 09 jan 1997.

BRIÃO, V. B.; TAVARES, C. R. G. Ultrafiltração como processo de tratamento para o reúso de efluentes de laticínios. Revista de Engenharia Sanitária e Ambiental, v. 12, n. 2, p. 134-138, 2007.

CAVAlCANTI, J. E. W. de A. Manual de tratamento de efluentes industriais. $2^{\mathrm{a}} \mathrm{ed}$. São Paulo: Engenho Editora Técnica, 2012. $453 p$. 
CHMIEL, H. et al. Reuse of vapour condensate from milk processing using nano filtration. Filtration+Separation, p. 24-27, 2000 .

CONSTANZI, R. N. Tratamento de efluentes domésticos por sistemas integrados de lodos ativados e membranas de ultrafiltração visando o reuso de água. 2007. 226 p. Tese (Doutorado em Engenharia Hidráulica) - Escola Politécnica de São Paulo, São Paulo, 2007.

GIACOBBO, A. et al. Microfiltração aplicada ao tratamento de efluentes de curtume. In: SIMPÓSIO INTERNACIONAL DE QUALIDADE AMBIENTAL, 7., 2010, Porto Alegre, Anais... Porto Alegre, Rio Grande do Sul, 2010.

HABERT, A. C. et al. Processos de separação por membranas. Rio de Janeiro: E-papers, 2006, 181 p.

HASAN, A. et al. A surface-renew all model of cross-flow microfiltration. Brazilian Journal of Chemical Engineering, v. 30, n. 1, p. 167-186, 2013.

HESPANHOL, I. Potencial de Reuso de Água no Brasil Agricultura, Indústria, Municípios, Recarga de Aquíferos. Revista Brasileira de Recursos Hídricos, v. 7, n. 4, p. 75-95, 2002.

KHIDER, K. A. et al. Purification of water effluent from a milk factory by ultrafiltration using Algerian clay support. Desalination, v. 167, p. 147-151, 2004.

LÁSLÓ, et al. Effect of preozonation on the filter ability of model dairy waste water in nanofiltration. Desalination, v. 240, p. 170177, 2009.
LAUTENSCHLAGER, R. S. et al. Modelação matemática e otimização operacional de processos de membrana de ultrafiltração. Revista de Engenharia Sanitária e Ambiental, v. 14, n. 2, p. 215-222, 2009.

LIMA, P. C. G. de. Uso dos mecanismos de detoxificação e tolerância aos metais cromo e cobre em Pseudokirchneriellasubcapitata e Pistiastratiotes e o uso das macrófitas Typhasp e Phragmitessp na remoção de nutrientes em wetlands construídos. 2010. 283 p. Tese (Doutorado em Engenharia Ambiental). Escola de Engenharia de São Carlos da Universidade de São Paulo, São Carlos, 2010.

MAGANHA, M. F. B. Guia técnico ambiental da indústria de produtos lácteos. São Paulo: CETESB, 2008. 95p.

MIERZWA, J. C.; HESPANHOL, I. Água na indústria: uso racional e reúso. São Paulo: Oficina de Textos, 2005. 143 p.

MIERZWA, J. C. Processos de separação por membranas: conceitos para uma aplicação eficiente. São Paulo, 2007. Disponível em: <http://www.cepema.usp.br/wp-content/ uploads/2011/06/IV-EPOA -4.pdf $>$. Acesso em: 15 mar. 2015.

PIRES, E. C. et al. Tratamento de águas residuárias industriais. In: CALIJURI, M. do C.; CUNHA, D. G. F. (coord). Engenharia ambiental: conceitos, tecnologia e gestão. Rio de Janeiro: Elsevier, 2013. 832 p.

SARAIVA, C. B. et al. Consumo de água e geração de efluentes em uma indústria de laticínios. Revista Instituto de Laticínios Cândido Tostes, v. 64, n. 367/368, p. 10-18, 2009.

SCHNEIDER, R. P.; TSUTYIA, M. T. Membranas Filtrantes para o tratamento de 
água, esgoto e água de reúso. São Paulo: ABES, 2001. 233 p.

SCHMITT, D. M. F. et al. Estudo da eficiência do composto ativo de Moringa Oleífera extraída com soluções salinas na tratabilidade de águas residuárias da indústria de laticínios. Revista Engevista, v. 16, n. 2, p. 221-231, 2014.

SCHIMITT, D. M. F. et al. Ultrafiltration combined with coagulation/flocculation/ sedimentation using Moringa oleifera as coagulant to treat dairy industry waste water. Water Air Soil Pollut, v. 224, n. 1682, p. 1-10, 2013.

SONG, L. Flux decline in cross flow microfiltration and ultrafiltration: mechanisms and modeling of membrane fouling. Journal of Membrane Science, v. 139, p. 183-200, 1998.
TCHAMANGO, S. et al. Treatment of dairy effluents by electro coagulation using aluminium electrodes. Science of the Total Environment, v. 408, p. 947-952, 2010.

TELLES, D.; D’COSTA, R. H. P. G. Reúso da água: conceitos, teorias e práticas. $2^{\mathrm{a}} \mathrm{ed}$. São Paulo: Blucher, 2010, 424 p.

VIDAL, C. M. de S.; CAMPOS, J. R. Coagulação associada à microfiltração para o tratamento avançado de esgoto sanitário. Revista Ambiência, v. 5, n. 1, p. 101, 2009.

VOURCH, M. et al. Treatment of dairy industry waste water by reverse osmosis for reuse water. Dessalination, v. 219, p. 190202, 2008.

WAGNER, J. Membrane Filtration Handbook: Practical Tips and Hints. $2^{\mathrm{a}} \mathrm{Ed}$. Osmonics, 2001. 129 p. 